\title{
Computational linguistics \& EFL reading comprehension: The KPG text classification profile
}

Trisevgeni Liontou

Greek Ministry of Education

https://doi.org/10.36505/ExLing-2015/06/0011/000248

\begin{abstract}
Advances in Computational Linguistics and Machine Learning systems have made it possible for EFL teachers, material developers and test designers to go beyond surface text components and adopt more theoretically sound approaches to text readability, focusing on a wider range of deep text features. Taking advantage of recent developments, the present research explored the existence of any statistically significant lexicogrammatical differences between intermediate and advanced reading comprehension exam texts of the Greek State Certificate of English Language Proficiency national exams in order to better define text complexity per level of competence. The main outcome of the research has been the Text Classification Profile that includes a qualitative and quantitative description features pertinent in intermediate and advanced reading comprehension exam texts.
\end{abstract}

Key words: linguistics, text complexity, reading comprehension

\section{Introduction}

The present study builds on earlier findings of research on reading assessment, according to which many text variables such as content, lexis and structure can have an impact on either the reading process or product and need to be taken into account during test design and validation (Oakland \& Lane 2004).). In fact, although a lot of research has been conducted in the field of second language acquisition with specific reference to ways of reading and text processing strategies, Alderson (2000) stressed language testers' lack of success "to clearly define what sort of text a learner of a given level of language ability might be expected to be able to read or define text difficulty in terms of what level of language ability a reader must have in order to understand a particular text". Such information would be particularly useful in providing empirical justification for the kinds of reading texts test-takers sitting for various language exams are expected to process, which to date have been arrived at mainly intuitively by various exam systems (Fulcher 1997).

\section{Aim of the study}

The aim of the present study was to delineate a range of linguistic features that characterize the reading comprehension texts used at the B2 (Independent

ExLing 2015: Proceedings of 6th Tutorial and Research Workshop on Experimental Linguistics, 26-27 June 2015, Athens, Greece 
User) and C1 (Advance User) level of the Greek national exams in English for the State Certificate of Language Proficiency -known with their Greek acronym as KPG exams- in order to better define text readability per level of competence and create a statistical model for assigning levels to texts in accordance with the purposes of the specific exam battery. In order to explore these issues, the following research question was formed:

1. Is there a specific set of text variables to better predict text difficulty variation between reading texts used at the $\mathrm{B} 2$ and $\mathrm{C} 1$ KPG English language exams?

\section{Methodology}

Over the last ten years, advances in Computational Linguistics and Machine Learning systems have made it possible to go beyond surface text components, focusing on a wider range of deep text features that take into account semantic interpretation and the construction of mental models and can, thus, offer a principled means for test providers and test-takers alike to assess this aspect of test construct validity (27). In the present study Coh-Metrix 2.1, Linguistic Inquiry and Word Count 2007 (LIWC), VocabProfile 3.0, Computerized Language Analysis (CLAN) suite of programs, Computerized Propositional Idea Density Rater 3.0 (CPIDR), Gramulator, Stylometrics and TextAnalyzer were used to estimate 135 text variables.

\section{Text Analysis Findings}

The ultimate purpose of the present research was the creation of a mathematical formula that could make possible the classification of English texts to two levels of language competence, that is, intermediate (B2) or advanced (C1), according to their level of linguistic complexity. In order to avoid contamination of results due to text length variation, the frequency counts of all indices were normalized to a text length of 100 words. IBM SPSS 20.0 statistical package data was used to compute descriptive statistics and perform reliability analyses, Pearson product moment correlations, T-tests, ANOVAs, multiple Linear and Binary Logistic Regressions.

In order to create our statistical model, regression analysis, a procedure commonly employed for predictive purposes, was used. The training set consisted of $63 \mathrm{KPG}$ reading texts, 34 of which had already been used in past B2 level exams and 29 in C1 level exams. These texts had originally been chosen as appropriate for each level of competence based on the judgement of experienced KPG test designers and a series of piloting sessions. Due to the high number of independent text variables and the two levels of text classification Binary Logistic Regression was carried out using the Forward method and the Wald criterion in IBM SPSS 20.0. Forward selection looks at 
each explanatory variable individually and selects the single explanatory variable that fits the data the best on its own as the first variable included in the model. Given the first variable, other variables are examined to see if they will add significantly to the overall fit of the model. Among the remaining variables, the one that adds the most is included. This latter step (examining remaining variables in light of those already in the model and adding those that add significantly to the overall fit) is repeated until none of the remaining variables would add significantly or there are no remaining variables (Cook et al. 2000).Following this method the program entered in the analysis one variable at a time, depending on whether it met a predefined set of statistical criteria $(p<0.05)$, and also removed those that contributed less to the predictive power of the model. To avoid over-fitting our model, a minimum of 15 cases of data for each predictor variable was considered acceptable, with the final model containing 4 predictor variables. Thus, with a ratio of 15.75 , the model followed the necessary statistical restrictions and distanced itself from over-fitting problems (Foster 2001). Based on the model, the resulting regression equation or prediction formula, which may provisionally be called the L.A.S.T. Text Difficulty Index is as follows:

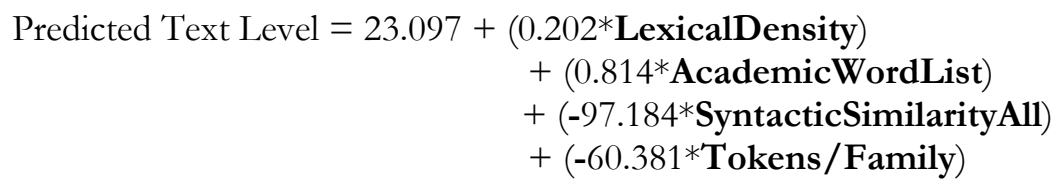

The specific regression model succeeded in correctly predicting the level of 33 of the 34 B2 texts $(97 \%)$ and 26 of the $29 \mathrm{C} 1$ texts $(90 \%)$. The total percentage of correct predictions on the training set was $94 \%$. This result signifies that the combination of the four variables alone managed to correctly classify 59 of the 63 B2 and C1 KPG reading texts used in the analysis. In other words, for the training set, using these specific four variables, the model has managed to correctly predict the level of $94 \%$ of the total number of preclassified passages. The Lexical Density (LD) variable refers to the proportion of content words to the total number of running words in a text estimated through LIWC2007, the Academic Word List variable relates to Coxhead's academic list and was estimated through VocabProfile, the Syntactic Structure Similarity variable for all sentences across paragraphs was calculated using CohMetrix 2.1, whereas the proportion of Tokens per Family was provided by VocabProfile. 


\section{Concluding remarks}

The preliminary results of the present study showed that the L.A.S.T. Text Difficulty Index could be used to draw a rough distinction between intermediate and advanced texts based on four linguistic features, i.e. lexical density, syntactic structure similarity, tokens per word family and technical vocabulary. This application might prove useful to test developers and other stakeholders interested in automatic text classification since texts calibrated to specific levels of language competence, can be fed into an electronic bank, from which test task writers of pen-and-paper or e-tests can more consistently choose source texts on the basis of specific text attributes. In addition, the proposed model might be of value in the context of classroom-based assessment, as well as for other exam batteries to better define what sort of text a reader of a prospective level of language ability should be able to process under real exam conditions. Undoubtedly, the new formula could be best viewed as a springboard for assessing text difficulty based on a more sound theory of language. For now, it remains to be explored whether the L.A.S.T. Text Difficulty Index will perform as well with a wider range of reading texts from various disciplines.

\section{References}

Alderson, C. 2000. Assessing Reading. Cambridge: Cambridge University Press.

Cook, P., Dixon, W., Duckworth, M., Kaiser, K., Koehler, W., Meeker \& Stephenson, W. (2000). Beyond Traditional Statistical Methods. Iowa: Iowa State University Press.

Foster, J. 2001. Data Analysis Using SPSS for Windows. London: Sage Publications Ltd.

Fulcher, G. 1997. Text difficulty and accessibility: Reading Formulas and expert judgment. System, 25, 4, 497-513.

Graesser, A., McNamara, D., Louwerse, M. \& Cai, Z. 2004. Coh-Metrix: Analysis of text on cohesion and language. Behavior Research Methods, Instruments \& Computers, 36, 2, 193-202. 